\title{
A Multi-Species Asymmetric Simple Exclusion Process and its Relation to Traffic Flow
}

\author{
V. Karimipour \\ School of Physics, \\ Sharif University of Technology \\ P.O.Box 11365-9161 \\ and \\ Institute for Studies in Theoretical Physics and Mathematics \\ P.O.Box 19395-5746 \\ Tehran, Iran
}

\begin{abstract}
Using the matrix product formalism we formulate a natural p-species generalization of the asymmetric simple exclusion process. In this model particles hop with their own specific rate and fast particles can overtake slow ones with a rate equal to their relative speed. We obtain the algebraic structure and study the properties of the representations in detail. The uncorrelated steady state for the open system is obtained and in the ( $p \longrightarrow \infty)$ limit, the dependence of its characteristics on the distribution of velocities is determined. It is shown that when the total arrival rate of particles exceeds a certain value, the density of the slowest particles rises abroptly.
\end{abstract}




\section{Introduction}

The Matrix Product Ansatz (MPA), first introduced in the work of Hakim and Nadal [1],become popular in the study of one dimensional stochastic exclusion models after Derrida et al [2] applied this technique to the simplest such model,namely the totally asymmetric simple exclusion process or ASEP[3]. For a recent review see [4]. They were able to find nontrivial representations for the relevant algebra and calculate many physical properties of this process. Since then this technique has been applied to many other interesting stochastic systems, both with periodic and with open boundary conditions. Janowsky and Lebowitz [5] have considered the case where there is a single impurity (a fixed blockage) on the lattice, where hopping rates are reduced, and have found that this single blockage has global effects, due to long range correlations. The case of a moving blockage and the formation of shocks has also been addressed in several works [6-7]. A model consisting of two species moving in opposite directions have been studied in Evans et al [8] where spontenous symmetry breaking has been observed. Finally MPA has been formulated for models with quasi parallel updatings [9-11].

In most of these works, a simplified model of traffic flow [12] is cited as a physical application, specially when impurities are present which when encountered decrease the rate of hopping of other particles [13]. Evans [14] has applied MPA to the case of several kinds of particles hopping with their own rates on a ring and has found that above a critical density a macroscopic number of holes are condensed in front of the slowest particle. In the work of Evans, however, particles can not overtake, and the order of particles is unchanged during the process.

Turning to the mathematical aspect of the problem, a matrix product state can be understood as a generalization of ordinary factorizable states with a product measure in which numbers are replaced with non-commuting objects. This allows the original problem to be reformulated in terms of algebraic relations. The main advantage of this thecnique is that once a nontrivial representation is found, all the physical quantities like the currents, densities and correlation functions can in principle be obtained from the representation. This is of course not a simple task and one should overcome many difficulties of combinatorial type in calculating explicit form of the matrix elements of product of operators.

Whether or not the zero energy state of a Hamiltonian with nearest neighbor interaction can always be formulated as a matrix product state has been answered in the affirmative by Sandow [15]. Thus to any Hamiltonian of the above type, corresponds an algebra in the MPA formalism. However finding nontrivial representions of this algebra may 
be harder than the original problem itself. Therefore some authors $[16,17]$ have tried to reverse the problem, thats to begin with a quadratic algebra,with hopefully simple representations and then to find the relevant stochastic process. In [17] the algebras considered are appearantly related to the partially assymetric case. This restriction has a two-fold disadvantage . First they obtain the concrete form of the algebraic relations only in cases for low number of species, and second they do not obtain [17] " a general framework for arbitrary $\mathrm{N}$ ( number of species ), which when specialized to $\mathrm{N}=2$ gives the know results."

Owr aim in this paper is to start from a suitable algebra and proceed to find a natural generalization of the one species ASEP.We will show that such a natural generalization exists. We will obtain the algebra, its representations and many of its properties. In this p-species ASEP each particle of type $\mathrm{j}$, hops with rate $v_{j}$ to its right neighbor site, and when it encounters a particle of type $i$ with $v_{j}>v_{i}$ they interchanges their site with rate $v_{j}-v_{i}$, as if the fast particle overtakes the slow one. In any other case the attempted move of the original particle is rejected. The model seems to be relevant as a simplified model of one way traffic flow. We consider open boundary conditions where particles ( or cars ) enter the system ( the highway ) with a rate proportional to their speed and leave at the other side.

The paper is organized as follows: In section 2 we introduce the algebra and solve its'consistency conditions. In section 3 we obtain the Hamiltonian which corresponds to this algebra. In section 4 we obtain the uncorrelated steady state and finally in section 5 we construct the infinite dimensioal representations of the algebra and present some of its useful properties. In all the steps we compare our resulsts with the one species ASEP.

\section{The Algebra}

We begin with an algebra generated by the elements $D_{i}, i=1, \ldots p$ and $E$, with the following relations:

$$
\begin{aligned}
D_{i} E & =\frac{1}{v_{i}} D_{i}+E \\
D_{j} D_{i} & =\xi_{j i} D_{j}+\eta_{j i} D_{i} \quad j>i
\end{aligned}
$$

where the parameteres $v_{i}$ are finite real numbers and the parameters $\xi_{j i}$ and $\eta_{j i}$ are to be determined. In writing $(1,2)$ we have had in mind a totally assymetric exclusion 
process.In order to be consistent with associativity we have to check that

$$
\left(D_{j} D_{i}\right) E=D_{j}\left(D_{i} E\right)
$$

Using (1) and (2), this requirement determines $\xi_{j i}$ and $\eta_{j i}$ as

$$
\xi_{j i}=\frac{v_{i}}{v_{i}-v_{j}} \quad \eta_{j i}=\frac{-v_{j}}{v_{i}-v_{j}}
$$

For the present we take all the $v_{i}$ to be different. Later we will relax this condition. We should also check that

$$
\left(D_{k} D_{j}\right) D_{i}=D_{k}\left(D_{j} D_{i}\right) \quad k>j>i
$$

Using (2) we find that this relation imposes no new relations on the parameters. Since any monomials of the form $D_{k} D_{j} \ldots D_{i}$ with $k>j>\ldots i$ can be reduced to a linear combination of generators, associativity is guaranteed for all the monomials of higher degree. The final form of the algebra reads

$$
\begin{aligned}
D_{i} E & =\frac{1}{v_{i}} D_{i}+E \\
D_{j} D_{i} & =\frac{1}{v_{i}-v_{j}}\left(v_{i} D_{j}-v_{j} D_{i}\right) \quad j>i
\end{aligned}
$$

where the parameters $v_{1} \ldots . v_{p}$ are free.

\section{The Hamiltonian and the Process}

We consider a one dimensional chain of $\mathrm{N}$ sites. The Hilbert space of each site is $p+1$ dimensional. The Hamiltonian is written as:

$$
H=h^{1}+H^{B}+H^{N}=h^{1}+\sum_{k=1}^{k=N-1} h_{k, k+1}^{B}+h^{N}
$$

Where $H^{B}$ is the bulk Hamiltonian and $h^{1}$ and $h^{N}$ are boundary terms. In the matrix product formalism, the steady state of this Hamiltonian can be written as:

$$
\mid P>=\langle W|\mathcal{D} \otimes \mathcal{D} \otimes \mathcal{D} \otimes \ldots \mathcal{D} \otimes \mathcal{D}| V>
$$


where $\mathcal{D}=\left(\begin{array}{l}E \\ D_{1} \\ \cdot \\ \cdot \\ D_{p}\end{array}\right)$ and $\mid V>$ and $<W \mid$ are vectors in an auxiliary space. According to MPA, we have $H \mid P>=0$ if the following relations are satisfied:

$$
\begin{gathered}
h^{B} \mathcal{D} \otimes \mathcal{D}=X \otimes \mathcal{D}-\mathcal{D} \otimes X \\
\left(h^{N} \mathcal{D}-X\right) \mid V>=0 \\
<W \mid\left(h^{1} \mathcal{D}+X\right)=0
\end{gathered}
$$

where we take $X$ to be $X=\left(\begin{array}{l}-1 \\ x_{1} \\ \cdot \\ \cdot \\ x_{p}\end{array}\right)$ with $x_{i}$ as c-numbers. Anticipating the process from the form of the algebra,we write the bulk Hamiltonian as follows:

$$
h^{B}=-\sum_{i=1}^{p} y_{i}\left(E_{0 i} \otimes E_{i 0}-E_{i i} \otimes E_{00}\right)-\sum_{j>i}^{p} y_{i j}\left(E_{i j} \otimes E_{j i}-E_{j j} \otimes E_{i i}\right)
$$

Here the matrices $E_{i j}$ act on the Hilbert space of one site and have the standard definition: $\left(E_{i j}\right)_{k, l}=\delta_{i k} \delta_{j l}$. The first term expresses an ASEP for each species of particles (i) with rate $y_{i}$. The second term represents an interchange of particles of type $(j>i)$, with rate $y_{i j}$. The parameters $y_{i}$ and $y_{i j}$ are to be determined.

The natural choice of the boundary terms should be such that particles of type $(i)$ are injected at the left and extracted at the right with their specific rates say $\alpha_{i}$ and $\gamma_{i}$ respectively. So we take:

$$
\begin{aligned}
& h^{1}=-\sum_{i=1}^{p} \alpha_{i}\left(E_{i 0}-E_{00}\right) \\
& h^{N}=-\sum_{i=1}^{p} \gamma_{i}\left(E_{0 i}-E_{i i}\right)
\end{aligned}
$$

Inserting (13) in (10) leads to the following equations:

$$
\begin{aligned}
\frac{y_{i}}{p} D_{i} E & =\frac{D_{i}}{p}+x_{i} E \\
\frac{y_{i j}}{p} D_{j} D_{i} & =x_{j} D_{i}-x_{i} D_{j}
\end{aligned}
$$


Comparision with (6) and (7) shows that:

$$
x_{i}=\frac{v_{i}}{p} \quad y_{i}=v_{i} \quad y_{i j}=v_{j}-v_{i}
$$

Inserting the values of the parameters $y_{i}$ and $y_{i j}$ in (13) clearly highlights the physical process governed by the Hamiltonian. Denoting a vacant site by the symbol $\phi$ and a site occupied by a particle of type i, by the symbol $A_{i}$, the process defined by $H^{B}$ is :

$$
\begin{aligned}
A_{i} \phi & \longrightarrow \phi A_{i} \quad \text { with rate } v_{i} \\
A_{j} A_{i} & \longrightarrow A_{i} A_{j} \quad j>i \text { with rate } v_{j}-v_{i}
\end{aligned}
$$

In order for all the rates to be positive we restrict the range of $v_{i}$ 's as:

$$
v_{1} \leq v_{2} \leq v_{3} \ldots \leq v_{p}
$$

Inserting (14) and (15) respectively in (11) and (12) we find the the following explicit matrix equations:

$$
\begin{aligned}
& \left.\left(\begin{array}{lllllll}
0 & -\gamma_{1} & -\gamma_{2} & -\gamma_{3} & . & . & -\gamma_{p} \\
0 & \gamma_{1} & 0 & 0 & . & . & 0 \\
0 & 0 & \gamma_{2} & . & . & . & 0 \\
0 & 0 & 0 & \gamma_{3} & . & . & 0 \\
. & \cdot & . & \cdot & . & . & . \\
. & . & . & . & . & . & . \\
0 & 0 & 0 & 0 & . & . & \gamma_{p}
\end{array}\right)\left(\begin{array}{l}
E \\
\frac{D_{1}}{p} \\
\frac{D_{2}}{p} \\
\cdot \\
\cdot \\
\cdot \\
\frac{D_{p}}{p}
\end{array}\right)-\left(\begin{array}{l}
-1 \\
\frac{v_{1}}{p} \\
\frac{v_{2}}{p} \\
\cdot \\
\cdot \\
\cdot \\
\frac{v_{p}}{p}
\end{array}\right)\right) \mid V>=0
\end{aligned}
$$

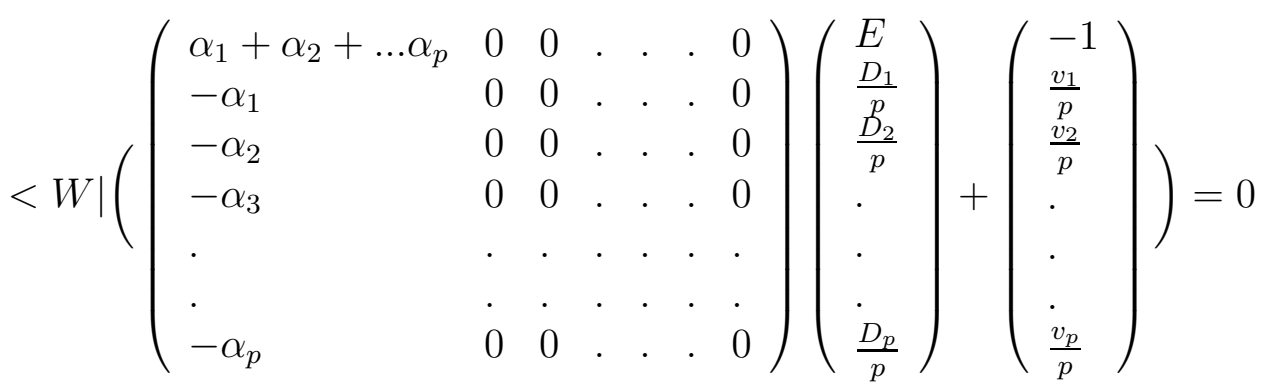

These equations then yield:

$$
\begin{aligned}
D_{i} \mid V> & =\frac{v_{i}}{\gamma_{i}} \mid V> \\
<W \mid E & =<W \mid \frac{v_{i}}{p \alpha_{i}}
\end{aligned}
$$


and

$$
\frac{v_{1}+v_{2}+\ldots v_{p}}{p}=1
$$

Equation (26) which means that the average speed of the particles is unity, is not really a condition on the rates, since by a rescaling of time one can always obtain this condition. Hereafter we will write this condition simply as $\langle v\rangle=1$. Equation (25) however implies that $\frac{v_{i}}{p \alpha_{i}}$ is independent of i. Thus we set $\alpha_{i}=\frac{1}{p} \alpha v_{i}$.

In order that equation (24) be consistent with (7), we require that :

$$
\left(D_{j} D_{i}-\frac{v_{i} D_{j}-v_{j} D_{i}}{v_{i}-v_{j}}\right) \mid V>=0
$$

After using (24) and rearranging terms, this condition shows that

$$
\gamma_{i}-v_{i}=\gamma_{j}-v_{j}
$$

Thus we set

$$
\gamma_{i}=v_{i}+\beta-1
$$

Therefore we have

$$
D_{i}\left|V>=\frac{v_{i}}{v_{i}+\beta-1}\right| V>\quad<W|E=<W| \frac{1}{\alpha}
$$

The meaning of the parameters $\alpha$ and $\beta$ are now clear. From (26) we find:

$$
\alpha_{1}+\alpha_{2}+\ldots \alpha_{p}=\alpha \quad \frac{\gamma_{1}+\gamma_{2}+\ldots \gamma_{p}}{p}=\beta
$$

Thus $\alpha$ and $\beta$ are respectively the total rate of injection and the average rate of extraction of particles. In order for the individual rates to be positive we restrict further the range of speeds as

$$
1-\beta \leq v_{1} \leq v_{2} \leq v_{3} \ldots \leq v_{p}
$$

The above results clearly justifies this model as a natural generalization of the 1-species ASEP.

We conclude this section with formulas for the current operators. A simple way to obtain these, is to directly refer to the process (19-20) and write the equation for the one point function $\left\langle\tau_{k}^{(i)}>\right.$ which is the average density of particles of type $(i)$ at site $\mathrm{k}$. 
Determining from (19-20) various ways of increasing and decreasing of this density and denoting the probabilty of site $k$ to be vacant by $\epsilon_{k}$ we find:

$$
\begin{aligned}
\frac{d}{d t}<\tau_{k}^{(i)}> & =v_{i}<\tau_{k-1}^{(i)} \epsilon_{k}>-v_{i}<\tau_{k}^{(i)} \epsilon_{k+1}> \\
& +\sum_{j<i}\left(v_{i}-v_{j}\right)<\tau_{k-1}^{(i)} \tau_{k}^{(j)}>-\sum_{j<i}\left(v_{i}-v_{j}\right)<\tau_{k}^{(i)} \tau_{k+1}^{(j)}> \\
& -\sum_{j>i}\left(v_{j}-v_{i}\right)<\tau_{k-1}^{(j)} \tau_{k}^{(i)}>+\sum_{j>i}\left(v_{j}-v_{i}\right)<\tau_{k}^{(j)} \tau_{k+1}^{(i)}>
\end{aligned}
$$

Note that inside any correclation function we have $\epsilon_{k}=1-\sum_{i} \tau_{k}^{(i)}$. Equation (30) can be rewritten as a continuity equation

$$
\frac{d}{d t}<\tau_{k}^{(i)}>=<J_{k}^{(i)}>-<J_{k+1}^{(i)}>
$$

where

$$
<J_{k}^{(i)}>=v_{i}<\tau_{k-1}^{(i)} \epsilon_{k}>+\sum_{j<i}\left(v_{i}-v_{j}\right)<\tau_{k-1}^{(i)} \tau_{k}^{(j)}>-\sum_{j>i}\left(v_{j}-v_{i}\right)<\tau_{k-1}^{(j)} \tau_{k}^{(i)}>
$$

According to MPA

$$
<J_{k}^{(i)}>=\frac{<W\left|C^{k-2} J^{(i)} C^{N-k}\right| V>}{<W\left|C^{N}\right| V>}
$$

where $C:=E+\frac{1}{p} D:=E+\frac{1}{p}\left(D_{1}+D_{2}+\ldots D_{p}\right)$. The current operators can be read from (31) to be:

$$
J^{(i)}=v_{i} \frac{D_{i}}{p} E+\sum_{j<i}\left(v_{i}-v_{j}\right) \frac{D_{i}}{p} \frac{D_{j}}{p}-\sum_{j>i}\left(v_{j}-v_{i}\right) \frac{D_{j}}{p} \frac{D_{i}}{p}
$$

Using eqs. $(6,7)$, we obtain:

$$
\begin{aligned}
J^{(i)} & =\frac{v_{i}}{p}\left(\frac{1}{v_{i}} D_{i}+E\right)-\frac{1}{p^{2}} \sum_{j<i}\left(v_{j} D_{i}-v_{i} D_{j}\right)-\frac{1}{p^{2}} \sum_{j>i}\left(v_{j} D_{i}-v_{i} D_{j}\right) \\
& =\frac{1}{p} D_{i}+\frac{v_{i}}{p} E-\frac{1}{p^{2}}\left(\sum_{j \neq i} v_{j}\right) D_{i}+\frac{v_{i}}{p^{2}}\left(\sum_{j \neq i} D_{j}\right) \\
& =\frac{1}{p} D_{i}+\frac{1}{p} v_{i} E-\frac{1}{p^{2}}\left(p-v_{i}\right) D_{i}+\frac{v_{i}}{p^{2}}\left(\frac{D}{p^{2}}-D_{i}\right) \\
& =\frac{v_{i}}{p} C
\end{aligned}
$$


We thus obtain

$$
<J^{(i)}>=\frac{v_{i}}{p} \frac{<W\left|C^{N-1}\right| V>}{<W\left|C^{N}\right| V>}
$$

Therefore all the currents are simply proportional to the average current $J$, however $J$ itself has a highly nontrivial dependence on the hopping rates.

In the next section we will find the one dimensional representations of the algebra. This case corresponds to the steady state being characterized by a Bernouli measure. Although very simple this steady state has a rather rich structure.

\section{One Dimensional Representations and the Uncor- related Steady State}

In the one dimensional representation the operators $D_{i}$ and $E$ are represented by cnumbers $\Delta_{i}$ and $e$ respectively. From equation (28) we have :

$$
\Delta_{i}=\frac{v_{i}}{v_{i}+\beta-1} \quad e=\frac{1}{\alpha}
$$

combining (35) and (6) gives the condition $\alpha+\beta=1$ on the average rates which is of the same form as in the one species asep. This, together with (29) means that for an uncorrelated steady state to exist, the minimum speed $v_{1}$ should be greater than the average arrival rate $\alpha$. The steady state is now given by $|P>=| \rho\rangle^{\otimes N}$ where

$$
\mid \rho>=\frac{1}{c}\left(\begin{array}{l}
e \\
\frac{\Delta_{1}}{p} \\
\frac{\Delta_{2}}{p} \\
\cdot \\
\cdot \\
\cdot \\
\frac{\Delta_{p}}{p}
\end{array}\right) \quad c=e+\frac{1}{p}\left(\Delta_{1}+\Delta_{2} \ldots \Delta_{p}\right) \equiv e+\frac{1}{p} \Delta
$$

The density and current of particles of type $(i)$ and the total density and the total current are all site independent and are respectively given by (see 34)

$$
\rho(\alpha, i)=\frac{\frac{\Delta_{i}}{p}}{e+\frac{\Delta}{p}} \quad J(\alpha, i)=\frac{\frac{v_{i}}{p}}{e+\frac{\Delta}{p}} \quad \rho(\alpha)=\frac{\frac{\Delta}{p}}{e+\frac{\Delta}{p}} \quad J(\alpha)=\frac{1}{e+\frac{\Delta}{p}}
$$


It is better to consider the limiting case $p \longrightarrow \infty$, that is, we assume that particle speeds are taken from a continous probability distribution $P(v)$. Condition (26) is then transformed into

$$
<v>:=\int v P(v) d v=1
$$

Discrete quantities $\frac{1}{p} f(i)$ are transformed into the continous functions $f(v) P(v)$ and sums into integrals. Instead of (36) we have

$$
\begin{array}{cr}
\rho(\alpha, v)=\frac{\Delta(\alpha, v) P(v)}{e+\Delta(\alpha)} & J(\alpha, v)=\frac{v P(v)}{e+\Delta(\alpha)} \\
\rho(\alpha)=\frac{\Delta(\alpha)}{e+\Delta(\alpha)} & J(\alpha)=\frac{1}{e+\Delta(\alpha)}
\end{array}
$$

where

$$
\Delta(\alpha, v)=\frac{v}{v-\alpha} \text { and } \Delta(\alpha)=\int \frac{v}{v-\alpha} P(v) d v
$$

As an example,in the following,we take a distribution which at low speeds vanishes as some power of $v-\alpha$, so that $\alpha$ is indeed the minimum speed of particles. Requiring that the distribution has an exponential decay rate $\lambda$ at high speeds and the average speed be unity gives:

$$
P_{\lambda}(v)=\frac{1}{\lambda^{m+1} \Gamma(m+1)}(v-\alpha)^{m} e^{\frac{-(v-\alpha)}{\lambda}}
$$

where $m+1=\frac{1-\alpha}{\lambda}$. Here $m$ is not necessarily an integer. A similar choice of $P(v)$ has also been made in [14]. Note that since $m$ is to be positive for each choice of $\lambda$, we should have $0<\alpha<1-\lambda$. This distribution is peaked at $v=1-\lambda$. Inserting (41) into (40) and doing the integral gives:

$$
\Delta(\alpha)=\frac{\lambda-1}{\lambda+\alpha-1}
$$

from which we obtain

$$
J(\alpha)=\frac{\alpha(\lambda+\alpha-1)}{\lambda-1+\alpha \lambda} \quad \rho(\alpha)=\frac{\alpha(\lambda-1)}{\lambda-1+\alpha \lambda}
$$

Eliminating $\alpha$ between the above equations gives the current versus density:

$$
J(\rho)=\rho\left(1-\frac{\rho}{1-\lambda(1-\rho)}\right)
$$

The curves $J(\alpha)$ and $J(\rho)$ are shown in figs.(1 and 2) for various values of the widths of the distributions. Note that for zero width $(\lambda=0)$ the familiar results of the one species 
ASEP are obtained, namely: $\rho=\alpha$ and $J=\rho(1-\rho)$. For any finite value of $\lambda \rho$ is an increasing function of $\alpha$ but $J(\alpha)$ has a maximum at $\tilde{\alpha}:=\frac{1-\lambda}{\lambda}(1-\sqrt{1-\lambda})$ The current at this optimal value of the arrival rate is

$$
J_{\max }(\lambda)=(1-\lambda)\left(\frac{1-\sqrt{1-\lambda}}{\lambda}\right)^{2}
$$

The maximum of $J$ as a function of $\rho$ occurs at $\tilde{\rho}=\frac{1}{\lambda}(\sqrt{1-\lambda}-(1-\lambda))$. It is seen that the maximum current has it is highest value of $\frac{1}{4}$ only when all the particles have the same hopping rates. It is interesting to note that even in such a simple uncorrleated steady state, a variance in hopping rates reduces the current.

The final quantity we consider is the average density of particles of different speeds, $\rho(\alpha, v)$ as a function of $v$. From (38-41) we have

$$
\rho(\alpha, v) \propto v(v-\alpha)^{m-1} e^{\frac{-(v-\alpha)}{\lambda}}
$$

The interesting point is that when $m \geq 1$ ( or $\alpha \leq 1-2 \lambda$ ) the density vanishes at the lowest speed $\alpha$ and has a maximum at $v_{\max }:=\frac{1}{2}\left(1-\lambda+\sqrt{(1-\lambda)^{2}-4 \lambda \alpha}\right)$. However when $m<1$ or $(\alpha>1-2 \lambda)$ the distribution changes and the density of the slowest particles rises abroptly. This transition is one of the interesting features of this process and is somehow reminicent of the Bose condensation first noted in [14].

\section{$5 \quad$ Representations}

As in the one-species ASEP the representations of this algebra are either one dimensional or infinite dimensional. To see this we first note that there is no non-zero eigenstate of $E$, say $\mid e>$ such taht $E\left|e>=\frac{1}{v_{i}}\right| e>$ for any $i$. Since if there is any such vector then by acting on both sides with $D_{i}$ and using (5) we obtain $\frac{1}{v_{i}} \mid e>=0$ and since $v_{i}$ is finite this means that $\mid e>=0$. Following [2] we know show that in any finite dimensional representation of the algebra all the generators mutually commute. For if the representation is finite dimensional then the matrices $E-\frac{1}{v_{1}}, \ldots E-\frac{1}{v_{p}}$ having no zero eigenvalue, are all invertible and hence (5) gives $D_{i}=E\left(E-\frac{1}{v_{i}}\right)^{-1}$ which in turn means that the representation is commutative. Thus the representations of this algebra are either one dimensional or infinite dimensional. Note that in the one dimensioanl case the expression $D_{i}=E\left(E-\frac{1}{v_{i}}\right)^{-1}$ automatically satisfy $(6,7)$. Thus such one dimensional 
representations really exist, and were used in section (4) to find the uncorrelated steady state.

To find the infinite dimensional representations we assume that there is one single vector denoted by $\mid 0>$ such that

$$
D_{i}\left|0>=d_{i}\right| 0>\quad i=1, \ldots p
$$

where the paramters $d_{i}$ are to be determined. We then consider the vector space $W$ spanned by the formal vectors $\left\{\left|n>:=E^{n}\right| 0>, n=0,1,2, \ldots\right\}$. Clearly

$$
E|n>=| n+1>, \quad \forall n
$$

Iterating (5) we find

$$
D_{i} E^{n}=v_{i}^{-n} D_{i}+v_{i}^{-n+1} E+v_{i}^{-n+2} E^{2}+\ldots v_{i}^{-1} E^{n-1}+E^{n}
$$

Thus we obtain:

$$
D_{i}\left|n>=v_{i}^{-n} d_{i}\right| 0>+v_{i}^{-n+1}\left|1>+v_{i}^{-n+2}\right| 2>+\ldots v_{i}^{-1}|n-1>+| n>
$$

In order to chech eq.(6) in this representation it is enough to consider only the state $\mid 0>$, since all the other states are obtained by acting on this state using the algebraic relations which have been found to be consistent. Therefore we require that

$$
\left(D_{j} D_{i}-\frac{1}{v_{i}-v_{j}}\left(v_{i} D_{j}-v_{j} D_{i}\right) \mid 0>=0\right.
$$

this fixes the parameters $d_{i}$ to be $d_{i}=\frac{v_{i}}{\epsilon+v_{i}}$ where $\epsilon$ is a constant independent of $i$. The explicit matrix form of the generators are then as follows:

$$
E=\left(\begin{array}{ccccccc}
0 & . & . & . & . & . & . \\
1 & 0 & . & . & . & . & . \\
. & 1 & 0 & . & . & . & . \\
. & . & 1 & 0 & . & . & . \\
. & . & . & . & . & . & . \\
. & . & . & . & . & . & . \\
. & . & . & . & . & . & .
\end{array}\right) \quad D_{i}=\left(\begin{array}{cccccccc}
d_{i} & \frac{d_{i}}{v_{i}} & \frac{d_{i}}{v_{i}^{2}} & \frac{d_{i}}{v_{i}^{3}} & \frac{d_{i}}{v_{i}^{4}} & . & . \\
0 & 1 & \frac{1}{v_{i}} & \frac{1}{v_{i}^{2}} & \frac{1}{v_{i}^{3}} & . & . \\
0 & 0 & 1 & \frac{1}{v_{i}} & \frac{1}{v_{i}^{2}} & . & . \\
. & . & . & . & . & . & . \\
. & . & . & . & . & . & . \\
. & . & . & . & . & . & . \\
. & . & . & . & . & . & .
\end{array}\right)
$$

\section{Remarks:}


1) If $\epsilon=0$,then all the $d_{i}=1$ and using (50) one sees that $D_{i} D_{j}=D_{j} D_{i} \quad \forall i, j$ although $D_{i} E \neq E D_{i}$. Hereafter we restrict ourselves this case. Note that this does not mean that the two point functions $\left\langle\tau_{k}^{(i)} \tau_{l}^{(j)}>\right.$ and $\left\langle\tau_{k}^{(j)} \tau_{l}^{(i)}>\right.$ are equal.

2) When $\epsilon=0$ we have $D_{i}=D_{j}$ for $v_{i}=v_{j}$, so that equation (7) becomes vaccuous in this case and no singularity arises due to $v_{i}=v_{j}$ in this equation. Furthermore one can safely eliminate one of these generators for the other one. This means that the (p-1)-ASEP algebra is naturally embedded in the (p)-ASEP algebra.

The ket and bra vectors $\mid V>$ and $<W \mid$ are found to be:

$$
\begin{aligned}
& \left|V>=\sum_{n=0}^{\infty}(1-\beta)^{n}\right| n> \\
& <W\left|=\sum_{n=0}^{\infty}(\alpha)^{-n}<n\right|
\end{aligned}
$$

Having a representation at hand is only half of the way in obtaining explicit expressions for the physical quantites like currents and correlation functions. To obtain these quantities,one should do lenghty calculations on the matrix elements of porduct of operators.Our work is not complete in the sence that we do not yet have the final explicit expression for these matrix elements. However we explore the properties of the representations as far as we can, hoping that using these, the rest of the problem be solved in another occasion, by this author or by others.

\subsection{Eigenvectors of $D_{i}$ 's}

Let $z$ be any complex number, with $|z|<v_{1}$. Define:

$$
\left|z>=\sum_{n=0}^{\infty} z^{n}\right| n>
$$

Then $\mid z>$ is a common eigenvector of all $D_{i}{ }^{\prime}$ 's:

$$
D_{i}\left|z>=\frac{v_{i}}{v_{i}-z}\right| z>
$$

This can be easily proved by direct calculation. The states $\mid z>$ can in fact be thought of as the coherent states of the algebra constructed as follows: $\left|z>:=\frac{1}{1-z E}\right| 0>$ where $\frac{1}{1-z E}$ is understood by its power series. We now define the dual vector $<z\left|=\sum_{n=0}^{\infty} z^{-n}<n\right|$. Then we have the following theorem, the proof of which is accomplished by noting that 
$<n|E=<n-1|$ and $<0 \mid E=0$, and doing straightforward calculations.

\section{Theorem:}

a) $<z\left|E=\frac{1}{z}<z\right|$

b) The coherent states form a basis of the space $\mathrm{W}$, and:

$$
\left|n>=\int_{|z| \leq 1} \frac{1}{\pi} d z d \bar{z} z^{-n}\right| z>
$$

c) The coherent states $\mid z>$ form an over complete basis for W. A complete basis is obtained by taking only the states with fixed $|z|$, with a completeness relation:

$$
1=\oint_{\text {fixed }|z|} \frac{d z}{2 \pi i z}|z><z|
$$

d) $<\omega \mid z>=\frac{1}{1-\frac{z}{\omega}}$ for $|z|<|\omega|$

\section{Remarks:}

1) It is now appropriate to recall that the states $|V\rangle$ and $\langle W|$ are in fact coherent states and so it is best to denote them respectively by $\mid 1-\beta>$ and $<\alpha \mid$.

2) The calculation of any physical quantity is now reduced to the calculation of matrix elements of powers of $C$ between the coherent states. For example, the average density $<n_{k}^{i}>$ is given as:

$$
<n_{k}^{i}>=\frac{1}{Z_{N}} \oint_{\left(1-\beta<|z|<\alpha, \quad|z|<v_{i}\right)} \frac{d z}{2 \pi i z}<\alpha\left|C^{k-1}\right| z><z\left|C^{N-k}\right| 1-\beta>\frac{v_{i}}{v_{i}-z}
$$

where $Z_{N}=<\alpha\left|C^{N}\right| 1-\beta>$. Or the probability of a segment [k,l] be empty is given by

$$
P(k, l):=\frac{1}{Z_{N}} \oint_{1-\beta<|z|<\alpha,} \frac{d z}{2 \pi i z}<\alpha\left|C^{k-1}\right| z><z\left|C^{N-k-l}\right| 1-\beta>\frac{1}{z^{l-k}}
$$

Once the eigenvalues and eigenvectors of $C$ are known,the problem can be solved completely. In the following we will give an implicit formula for these objects. 


\subsection{Eigenvalues of $\mathrm{C}$}

First we consider the case $p=1$. For any coherent state $\mid z>$ we have $E \mid z>=\frac{1}{z}(\mid z>$ $-\mid 0>)$. For any two complex numbers $z$ and $\omega$ define:

$$
|z, \omega>=z| z>-\omega \mid \omega>
$$

Then it follows that:

$$
E|z, \omega>=| z>-\mid \omega>
$$

Since for $p=1$ we have $D\left|z>=\frac{1}{1-z}\right| z>$ we obtain:

$$
C|z, \omega>=(D+E)| z, \omega>=\frac{1}{1-z}\left|z>-\frac{1}{1-\omega}\right| \omega>
$$

Therefore the state $\mid z, \omega>$ is an eigenstate of $C$ if we have $z(1-z)=\omega(1-\omega)$, the solution of which is $\omega=z$ or $\omega=1-z$ The second solution is acceptable and hence we have:

$$
C\left|z, 1-z>=\frac{1}{z(1-z)}\right| z, 1-z>
$$

Now let $p>1$. Using (56) and (60), we obtain:

$$
C|z, \omega>=z \eta(z)| z>-\omega \eta(\omega) \mid \omega>
$$

where

$$
\eta(z)=\frac{1}{z}+\frac{1}{p} \sum_{i=1}^{p} \frac{v_{i}}{v_{i}-z}
$$

The state $\mid z, \omega>$ is an eigenvector of $C$ if $z$ and $w$ lie on the curve $\eta(z)=\eta(\omega)$. We will then have: $C|z, \omega(z)>=\eta(z)| z, \omega(z)>$.

Note that in the $p \longrightarrow \infty z$ and $\omega$ are related by the following equation:

$$
\frac{1}{z}+\int \frac{v P(v)}{v-z}=\frac{1}{\omega}+\int \frac{v P(v)}{v-\omega}
$$

In order to understand the connection with the eigenstate obtained in Derrida et al [2] in the $p=1$ case we change the basis of $\mathrm{W}$ as:

$$
\left|e_{n}>=(E-1)^{n}\right| 0>, \quad\left|e_{n}>=\right| 0>
$$

Using ( 6,7$)$ it is easily found that:

$$
E\left|e_{n}>=\right| e_{n}>+\mid e_{n+1}>
$$




$$
\begin{gathered}
D\left|e_{n}>=\right| e_{n}>+\mid e_{n-1}> \\
C\left|e_{n}>=\right| e_{n-1}>+2\left|e_{n}>+\right| e_{n+1}>
\end{gathered}
$$

Thus we have

$$
\left|z>=\frac{1}{1-z E}\right| 0>=\frac{1}{1-z-z(E-1)}\left|0>=\frac{1}{1-z}\left(\frac{1}{1-\frac{z}{1-z} E}\right)\right| 0>=\frac{1}{1-z} \mid \frac{z}{1-z}>_{d}
$$

where by $\mid z>_{d}$ we mean $\left|z>_{d}=\sum_{n=0}^{\infty} z^{n}\right| e_{n}>$ Therefore

$$
\left|z, 1-z>=\frac{z}{1-z}\right| \frac{z}{1-z}>_{d}-\frac{1-z}{z}\left|\frac{1-z}{z}>_{d}=\sum_{n=0}^{\infty}\left(\left(\frac{z}{1-z}\right)^{n+1}-\left(\frac{1-z}{z}\right)^{n+1}\right)\right| e_{n}>
$$

Taking $\frac{z}{1-z}=e^{i \theta}$ gives

$$
\left|z, 1-z>=\sum_{n=0}^{\infty} \sin (n+1) \theta\right| e_{n}>
$$

which is denoted by $\mid \theta>$ in [2] with eigenvalue $\frac{1}{z(1-z)}=2(1+\cos \theta)$. This concludes our treatment of the representations of the algebra.

\section{Discussion}

This work can be pursued further in the following directions:

a) Finding a solution of the equation $\eta(z)=\eta(\omega)$ either for a low value of $p(e \cdot g \cdot p=2)$ or in the $p \longrightarrow \infty$ limit, and then expanding the state $\mid 1-\beta>$ in terms of the eigenstates of $C$. In this way one will obtain the current and the phase diagram of the system.

b) Finding a solution of the mean field equations, either numerically or analytically. These equations can be written down from (31). In the $p \longrightarrow \infty$ limit they are:

$$
\alpha v e_{1}=J_{k}(v)=J_{k+1}(v)=\ldots=(v+\beta-1) n_{N}(v)
$$

where

$$
J_{k}(v)=v n_{k}(v) e_{k+1}+\int_{0}^{v}\left(v-v^{\prime}\right) n_{k}(v) n_{k+1}\left(v^{\prime}\right) d v^{\prime}-\int_{v}^{\infty}\left(v^{\prime}-v\right) n_{k}\left(v^{\prime}\right) n_{k+1}(v) d v^{\prime}
$$

Here $n_{k}(v)$ is average density of particles of speeds $v$ at site $k$ and $e_{k}:=1-\int_{0}^{\infty} n_{k}(v) d v$ is the probability of site $k$ being vacant. In the one species model it is known that the mean field analysis gives the phase diagram correctly. So it will be interesting to see for 
a typical probability distribution how the phase diagram will be modified.

\section{Acknowledgements}

My Special Thanks go to A.Langari for helping me in drawing the figures. I would also like to thank R. Ejtehadi, J. Davoodi,and M.R. Rahimitabar for stimulating discussions, and F. Jafarpour, M. E. Fouladvand and A. Shafi Dehabad for valuable comments. 


\section{Figure Captions}

Figure 1: The current versus the arrival rate of particles for different values of $\lambda$. Figure 2: The current versus the density for different widths of the distribution. 


\section{References}

1. Hakim V and Nadal J P 1983 J.Phys. A ; Math. Gen. 16 L213

2. Derrida B Evans M R Hakim V and Pasquier V 1993 J.Phys. A ; Math. Gen. 261493

3. Liggett T M 1985 Interacting Particle Systems ( New York; Springer)

4. Derrida B 1998 Phys. Rep. bf 30165

5. Janowsky S A and Lebowitz J L 1994 J. Stat. Phys. 7735 Janowsky S A and Lebowitz J L 1992 Phys. Rev. A 45618

6. Derrida B Janowsky S A Lebowitz J L and Speear E R 1993 Europhys . Lett.22 651

Derrida B Janowsky S A Lebowitz J L and Speear E R 1993 J. Stat. Phys.73 831

7. Mallik K 1996 J. Phys. A ; Math. Gen. 295375

8. Evans M R Foster D P Godreche C and Mukamel D 1995 J. Stat. Phys. 80 ; Phys. Rev. Lett.74,208(1995).

9. Hinrichsen H 1996 J.Phys. A ; Math. Gen. 293659

10. Rajewsky N Schadschneider A and Schreckenberg M 1996 J.Phys. A ; Math. Gen. 29 L305

11. Honecker A and Peschel I 1996 J.Stat. Phys. 88319

12. Schreckenberg M Schadschneider A Nagel K and Ito M 1995 Phys. Rev. E 512339

13. Lee H W Popkov V and Kim D 1997 J.Phys. A ; Math. Gen. 30 8497 
14. Evans M R 1996 Europhys. Lett. 3613

15. Kreb K and Sandow S 1997 J.Phys. A ; Math. Gen. 303165 Stochastic Systems and Quantum Spin Chains preprint cond-mat/9610029

16. Arndt P Heinzel T and Rittenberg V, 1998 J.Phys. A ; Math. Gen. 31833

17. Alcaraz F C Dasmahapatra S and Rittenberg V, 1998 J.Phys. A ; Math. Gen. 31845 


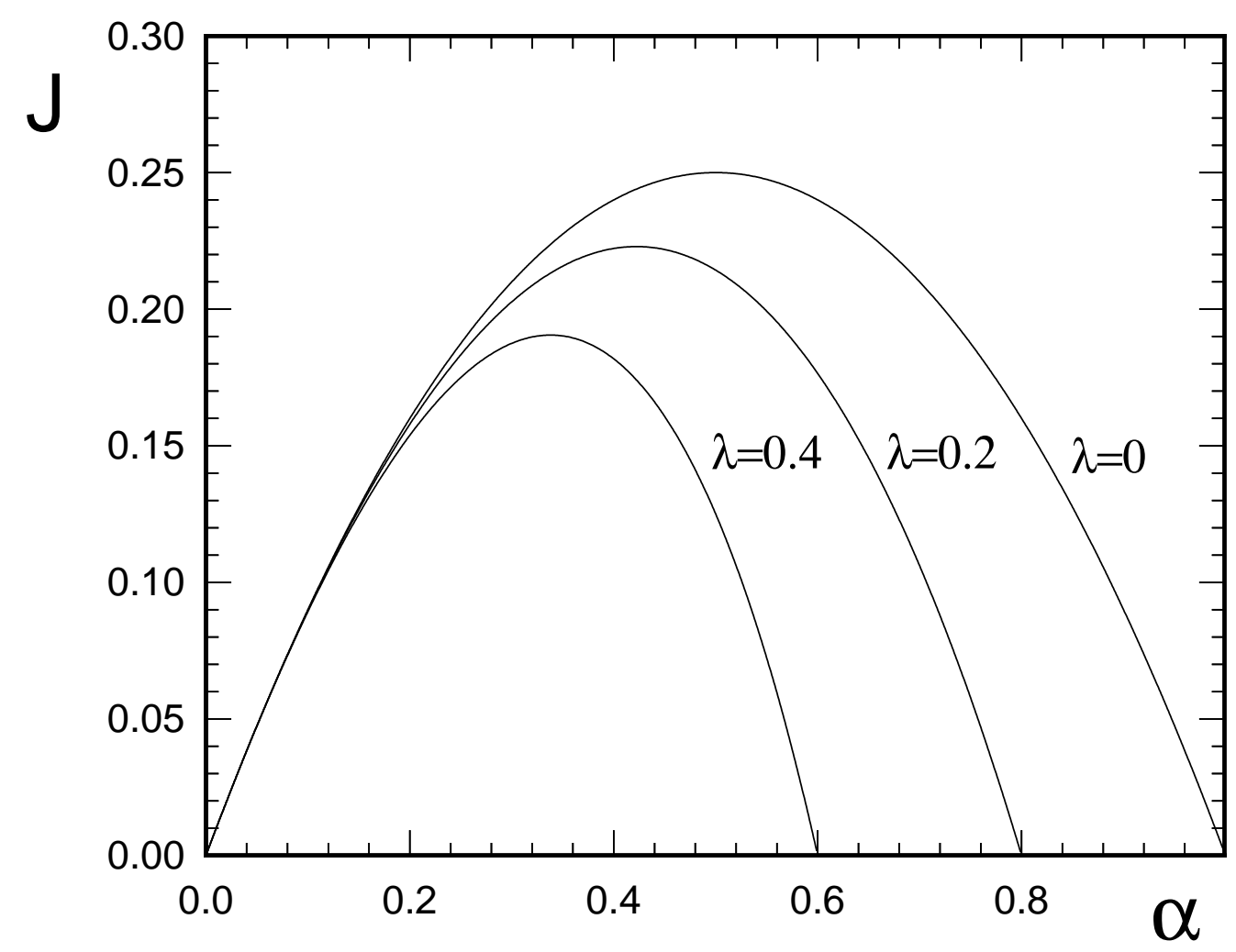

Figure.1 


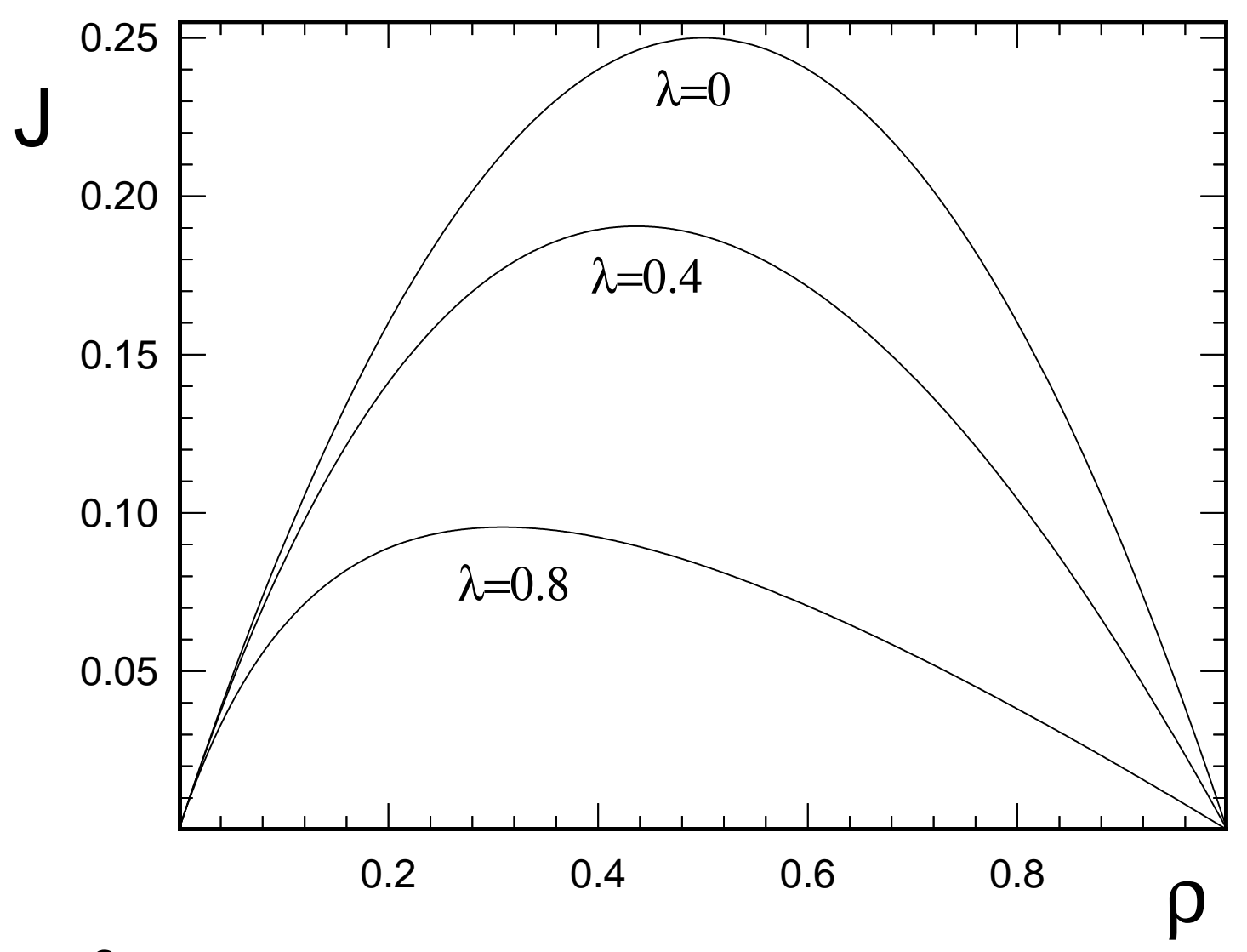

Figure.2 